\title{
Kyste dysembryologique médian dorsolingual : \\ à propos d'un cas
}

\section{Dorso-lingual median cyst of developmental origin: a case report}

PHILIPPE CAMPAN*, PHILIPPE KEMOUN*,LISON PESSINET-BENOUAICH**, MARTINE NESPOULOUS**

\section{RÉSUMÉ}

Un adolescent de 14 ans présente à l'examen clinique une formation dorso-linguale de type tumorale bénigne, dont l'antériorité permet de mettre en évidence une certaine stabilité. Aucune anomalie collatérale n'est objectivable notamment au niveau thyroïdien. Une biopsie-exerèse de la formation linguale est réalisée. L'examen histopathologique montre la présence d'une formation épithéliale vestigiale, circonscrivant une cavité quasi-virtuelle au sein de la tumeur, évoquant une formation kystique dégénérée. Le diagnostic de kyste lingual médian d'origine embryologique est alors proposé et discuté en fonction des données de la littérature. II en résulte que le kyste lingual médian n'apparaît pas comme une entité nosologique homogène, mais semble regrouper plusieurs types de dysembryoplasies kystiques dorso-linguales médianes, polymorphes, tant d'un point de vue clinique que histopathologique (Med Buccale Chir B uccale 2002; 8: 53-7).

mots clés : Kystes et tumeurs linguaux, Kystes du tractus thyréoglosse

médecine buccale chirurgie buccale VOL. $8, \mathrm{~N}^{\circ}$ 2002 page 53

\section{SUMMARY}

This study, based on the clinical examination of a 14-year-old adolescent, revealed a benign dorso-lingual tumor whose length of diachronic evolution showed a certain stability. No evidence of collateral abnormality particularly in respect to the thyroid was found. A biopsy-excision was performed. The histopathological examination showed the presence of a vestigial epithelial formation, surrounding a virtual cavity within the tumor, consistent with a degenerated cystic formation. The diagnosis of a median lingual cyst of embryological origin was then suggested and discussed according to the literature. From both clinical and histopathological standpoint, median lingual cyst does not appear as a nosological homogenous entity, but seems to group several types of polymorphous median dorso-lingual cystic dysembryoplasia. (Med Buccale Chir Buccale 2002; 8: 53-7).

key words : Oral Cyst, Embryopathies, Tongue Disease, Thyroglossal Cyst

* U.F.R d'Odontologie. TOULOUSE. France

**Anatomo-Pathologistes 116, bd Déodat-de-Sévérac 31300 TOULOUSE. France

Demande de tirés à part :

UFR odontologie 3 chemins des Maraîchers 31400 Toulouse

Article reçu le 12 octobre 2000. Accepté pour publication le 29 novembre 2001 
médecine buccale chirurgie buccale

L. $8, \mathrm{~N}^{\circ} 1$

2002

page 54
Les kystes et les fistules d'origine embryonnaire de la face et du cou sont des anomalies peu fréquentes représentant environ $2 \%$ des tumeurs cervicales [1]. Parfois découverts à l'âge adulte, ils sont le plus souvent révélés pendant l'enfance. Ainsi, la régression incomplète d'une structure embryonnaire ou la migration anormale d'un organe peuvent être à l'origine d'anomalies diverses : branchiales (kystes branchiaux), du tractus thyréoglosse (kyste du tractus thyréoglosse (TTG)), de l'angiogénèse (hémangiome, lymphangiome), du développement tissulaire avec inclusions de débris épithéliaux (tératomes, kystes dermoïdes et épidermoïdes, kystes médio-linguaux). [2-4]

La diversité des dysembryoplasies rend l'interrogatoire, la clinique et les différents examens radiographiques souvent insuffisants pour identifier la lésion. L'anatomopathologie permet le plus souvent d'établir un diagnostic, mais les remaniements tissulaires post-nataux des structures en cause le rendent parfois difficile à affirmer.

\section{OBSERVATION}

Un adolescent de 14 ans vient consulter pour une tuméfaction située sur la face dorsale de la langue, en position médiane, juste en avant du foramen caecum (Fig.1).

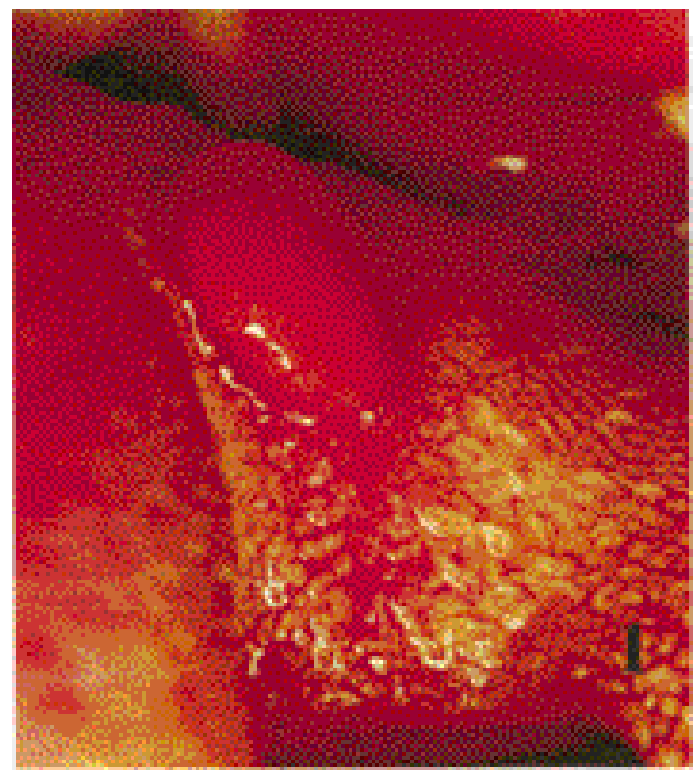

Figure 1 : Aspect clinique de la lésion Clinical aspect of the lesion
Il s'agit d'une masse sessile, arrondie, d'un centimètre de diamètre, faisant saillie sur le dos de la langue, indolore, bien limitée, ferme à la palpation et de consistance plutôt élastique. Sa surface est dépapillée, de couleur rose, sans ulcération, ni plage de kératinisation, ni fistule.

Aucune autre lésion linguale ou pelvilinguale n'est retrouvée. Le patient présente cependant une hyperplasie gingivale localisée à la région antérieure, d'étiologie traumatique, liée à une supraclusie incisive en cours de réduction par un traitement orthodontique. II n'y a pas d'adénopathie cervico-faciale, ni de tuméfaction visible ou palpable. Aucune gêne fonctionnelle n'est décrite, et à l'examen, la protraction de la langue est normale. Observée déjà 6 ans auparavant à l'occasion d'une adénoïdectomie, l'évolution de la tuméfaction semble stable. A l'interrogatoire, il n'est pas retrouvé d'antécédent notable: pas de dysthyroïdie, pas de traumatisme lingual, pas de lésion kystique ou nodulaire cervico-faciale ou une autre localisation.

Les signes cliniques sont en faveur de la bénignité de cette lésion : unique, topographie bien circonscrite, petite taille, stabilité, absence d'ulcération, d'épaississement muqueux et de saignement, pas d'adénopathie satellite. Ceci n'a motivé ni examen radiologique complémentaire (échographie, scannographie, IRM, scintigraphie), ni exploration ORL ou endocrinienne.

Le diagnostic clinique de kyste lingual médian d'origine embryonnaire est alors évoqué, et son exérèse chirurgicale préconisée.

Compte tenu de la faible accessibilité de la tumeur, de par sa situation linguale à la lisière du V lingual, l'intervention est conduite sous anes-thésie générale, avec intubation naso-trachéale. Le dôme de la tuméfaction est maintenu par une pince à forcipressure, sa base est incisée au bistouri en direction apicale. Le clivage dans sa portion inférieure s'avérant impossible, une dissection prudente en quartier d'orange est pratiquée. Une suture point par point aux plans profond et superficiel, ferme la plaie et permet d'obtenir l'hémostase.

L'examen histologique montre l'existence au centre de la lésion d'une formation kystique, limitée par un épithélium malpighien kératinisé, localement mono ou bi-stratifié. Autour de ce kyste, le stroma 
périphérique, très richement vascularisé, prend un aspect pseudo-angiomateux et les fibres musculaires, de par leur disposition et leur regroupement, semblent s'organiser. II n'y a aucun élément à caractère suspect de malignité, ni aucune atteinte de type infectieuse ou inflammatoire. L'origine dysembryologique de ce kyste est vraisemblable (Fig. 2 et 3). Les suites opératoires sont simples et la consultation à un mois montre une cicatrisation totale.

\section{COMMENTAIRES}

Chez un enfant ou un adolescent, face à un syndrome de masse dorso-lingual médian, d'évolution lente, dans un contexte non infectieux, sans symptomatologie bruyante et sans signe clinique de malignité, le diagnostic de kyste d'origine dysembryologique semble être le plus envisageable.

II s'agit de développements cavitaires consécutifs à l'inclusion et/ou à l'absence de résorption de débris tissulaires lors de l'embryogénèse. L'origine de ces tissus détermine le type de kyste en présence. La revue de la littérature fournit un grand nombre de publications rapportant des lésions kystiques linguales médianes [1-11].

Bien qu'aucune statistique ne soit disponible, il semble que les anomalies les plus fréquentes soient les kystes du tractus thyréoglosse (KKT). Le tractus thyréoglosse (TTG) est un canal épithélial, laissé «en traînée» par la thyroïde depuis
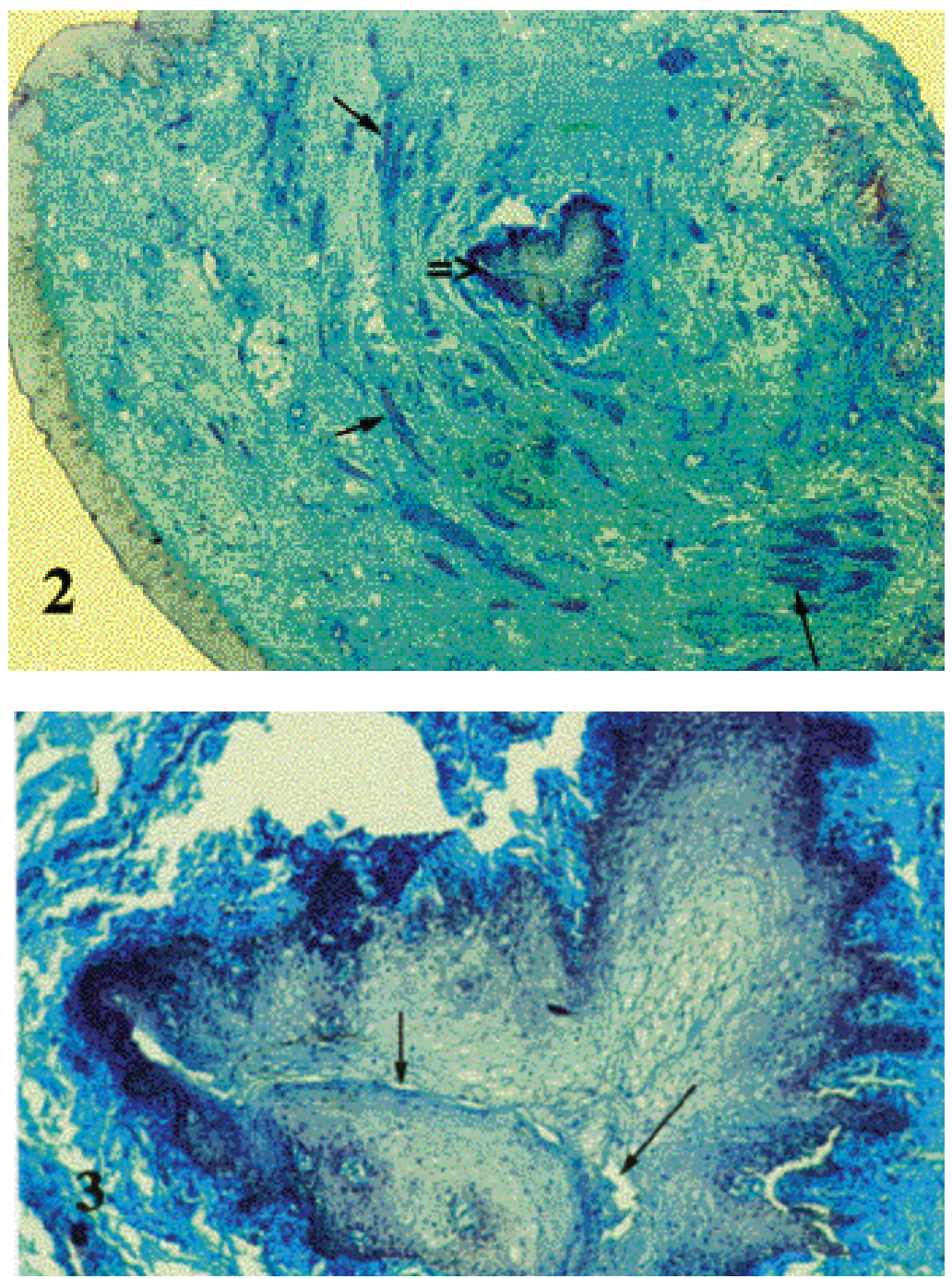

Figure 2 : Aspect histologique de la lésion (x25). Noter l'organisation concentrique des faisceaux musculaires lisses $(\longleftarrow$ ) autour de la formation épithéliale vestigiale $(\longleftarrow)$. Histological aspect of the lesion (x25). Note the concentric disposition of the smooth muscular fibers $\Longrightarrow$ ) surrounding the vestigial epithelial formation $(\Longrightarrow$ ).

Figure 3 : Aspect histologique de la lésion (x100). Noter la cavité kystique vestigiale. $(\longleftarrow)$

Histological aspect of the lesion $(x 100)$. Note the vestigial cystic cavity $(\longleftarrow)$ médecine buccale chirurgie buccale VOL. $8, \mathrm{~N}^{\circ}$ 2002 page 55 
médecine buccale chirurgie buccale

L. $8, \mathrm{~N}^{\circ} 1$

2002

page 56 son site lingual de formation, à la pointe $d u \mathrm{~V}$ lingual [12] à la cinquième semaine du développement embryonnaire, jusqu'en avant du larynx vers la septième semaine. Normalement, le TTG doit involuer complètement. Quand il ne disparaît pas, les reliquats, consécutifs à la présence de restes de ce conduit peuvent prendre l'aspect d'une «thyroïde linguale » ou exceptionnellement la forme d'un canal vestigial dit «canal de Bochdalek» mais le plus souvent l'aspect de nodules ou de kystes thyréoglosses isolés [17]. Ces kystes peuvent siéger n'importe où sur son trajet, sur la ligne médiane du cou, ou à son voisinage immédiat. La localisation linguale représente $2,1 \%$ de ces formations [6]. D'un point de vue histopathologique, on retrouve une cavité kystique vraie, bordée d'un épithélium pavimenteux stratifié kératinisé, avec des plages d'épithélia pseudo-stratifiés ciliés, de type respiratoire ou des cellules thyroïdiennes bordant le kyste.

La particularité de la lésion observée est qu'elle renferme une formation épithéliale avec une lumière très fine, le tout évoquant une cavité kystique vestigiale. L'épithélium kystique est un épithélium malpighien mono ou bistratifié avec une couche cornée ayant produit localement de la kératine. Cependant, il n'est retrouvé ni épithélium de type cilié ni cellules thyrö̈diennes. II n'est donc pas possible d'affirmer qu'il s'agit d'un KTT.

La seconde entité envisageable est le kyste épidermoïde. II s'agit d'une lésion assez rare, du tiers antérieur de la langue, souvent diagnostiquée entre 12 et 15 ans. Elle peut ressembler à la lésion que nous rapportons, bien qu'Akerson [5] décrive une tuméfaction prenant l'ensemble du dos de la langue, assez mal limitée et non dépapillée. L'étiopathogénie s'explique par l'inclusion et l'absence de résorption du mur ectodermique lors de la fusion des bourgeons latéro-linguaux à la sixième semaine du développement intra-utérin. Microscopiquement, on retrouve un épithélium stratifié kératinisé [13].

Le troisième diagnostic à envisager, est le kyste lingual médian, qui semblerait le plus correspondre au contexte. Cliniquement, des descriptions assez divergentes sont retrouvées. Certains auteurs décrivent des tuméfactions sessiles de l'ensemble du dos de la langue, avec une muqueuse linguale de recouvrement normale [2]. D'autres rapportent des lésions plus circonscrites, avec une muqueuse linguale dépapillée, cliniquement beaucoup plus proches de celle du cas que nous évoquons [3], mais dont la localisation concerne le tiers lingual antérieur. Histologiquement, on retrouve la prédominance d'un épithélium pseudo-stratifié cilié typique des structures respiratoires. II s'agirait de l'inclusion d'une partie du bourgeon de l'arbre respiratoire dans les bourgeons linguaux, phénomène facilité par la contiguité des deux systèmes embryonnaires. Pourtant, l'aspect nosologique de cette entité est souvent remis en question [3-4]. En effet, on retrouve différents diagnostics, kyste linguaux d'origine respiratoire, glossocèle, kyste intra-lingual d'origine respiratoire, kyste muqueux, etc. (Quinn, Fink, Samuel, Constantinides, rapportés par Naidoo [3]), consacrés à ces formations lésionnelles dorso-linguales dont l'histopathologie révèle indifféremment des épithéliums kystiques d'allure gastrique (épithélium cuboïde), stratifiés kératinisés, pseudo-stratifiés ciliés, solitaires ou associés. C'est la raison pour laquelle Naidoo [3] propose le terme de kyste lingual médian dans tous les cas.

Les autres diagnostics tels que les kystes latéraux du cou, les lymphangiomes ou les processus infectieux sont facilement écartés par l'examen clinique.

L'anatomo-pathologie et l'imagerie permettent également d'éliminer :

- la thyroïde linguale, de par les îlots thyroïdiens facilement reconnaissables sur une coupe, la palpation cervicale thyroïdienne négative, la TDM linguale mettant en évidence une hyperdensité basi-linguale, et la scintigraphie fixant le technétium au niveau basi-lingual et non au niveau sus-sternal $[9,14,15]$;

- les tératomes, de par la présence de tissus mésodermiques et entoblastiques (digestifs) et sa prédominance chez le nouveau-né [16] ;

- le kyste dermoïde lingual, de par sa localisation préférentielle sublinguale et la présence d'annexes de la peau [8] ;

- le lipome qui contient des adipocytes. 
Il n'est pas retrouvé dans la littérature de cas tel que celui décrit dans cette observation. Compte tenu de l'examen clinique et microscopique, il est difficile de retenir un des diagnostics précités, mais il semble judicieux de classer cette lésion parmi les kystes linguaux médian d'origine embryonnaire. La cavité kystique existe, mais l'identification des vestiges épithéliaux est difficile. En l'absence d'anticorps spécifiques, l'immunohisto-

\section{RÉFÉRENCES}

1- Mauruc B, Faugère J M, Douce P, Gouteyron J F. Kyste du tractus thyréoglosse à localisation basilinguale. Rev Laryngo 1992; 113: 205-6.

2- Manor Y, Buchner A, Peleg M, Taicher S. Lingual cyst with respiratory epithelium: an entity of debatable histogenesis. J Oral Maxillofac Surg 1999; 57: 124-7.

3 - NAIDOO L. Median lingual cyst: review of the literature and report of a case. J Oral Maxillofac Surg 1997; 55: 172-5.

4 - Wiersma R, Hadley GP, Bosenberg at, Chrystal V. Intralingual cysts of foregut origin. J Pediatric Surg 1992; 27: 1404-6.

5 - AKerson H, Milford M. Epidermoid cyst of the tongue: report of case. J Oral Surg 1974; 32: 117-20.

6 - Allard R. The thyroglossal cyst. Head Neck Surg 1982; 5: 134-46.

7 - Fontaine P, Truy E, Kauffmann I, Disant F, Morgon A. Kystes et fistules congénitaux de la face et du cou. Pédiatrie 1992; 47: 617-22.

8 - Gold D, Sheinkopf D, LeVY B. Dermoid, epidermoid, and teratomatous cysts of the tongue and the floor of the mouth. J Oral Surg 1974; 32: 107-11.

9 - Marsot-Dupuch K, Levret N, Pharaboz C, Robert $Y$, El Maleh M, Meriot P, Poncet J L, Chabolle F. Pathologie cervicale congénitale, origine embryologique et diagnostic. Rapport Du Cireol. J Radiol 1995; 76: 405-15. chimie ne peut être utilisée afin d'identifier un épithélium vestigial. De plus, il est cependant intéressant de remarquer l'organisation périvestigiale de la lésion, qui pourrait s'expliquer par une tentative de circonscription des vestiges, tel un homéocontrôle. La vascularisation importante et l'organisation concentrique des fibres musculaires viennent conforter l'origine dysembryologique de la lésion.

10 - Reddy VS, Radhakrishna K, Rao PLN. Lingual dermoid. J Pediatric Surg 1991; 26: 1389-90.

11 - Tsang-Pal Liu, Kuo-Shyang J eng, Tsen-long Yang, Teh-Ching Wang, Kong-Fant Hwang. Thyroglossal duct cyst: an analysis of 92 cases. Chin Med J 1992; 49: 72-5.

12- LARSEN W.J. Embryologie Humaine (pp 334). De Boeck University, Bruxelles,1996.

13 - Gorlin JR, Goldman HM, Thoma's Oral Pathology (pp 463-4) - MOSBY- Vol.1. $6^{\circ}$ ed. St Louis, 1970.

14 - Shafer WG, Maynard KH, B arnet ML. A textbook of oral pathology (pp 28) - $3^{\circ}$ ed. illustrated. W.B Saunders Compagny, Philadelphia,1974.

15 - Guerin W, Urtasun A, Chauveau E, Julien $M$, Lebreton M, Dumon M. Thyrö̈de linguale et kyste du tractus thyréoglosse basi-lingual. A propos de 2 cas. Rev Laryngol Otol Rhinol 1997; 118: 183-8.

16 - LAWLANI AK, ENGEL TL. Teratoma of the tongue: a case report and review of the literature. Int J Pediatr Otorhinolaryngol 1992; 24: 261-8.

17 - Ortonne J P. Pathologie de la pigmentation cutanéomuqueuse (pp. 609-972) In : Traité de pathologies buccale et maxillo-faciale. Piette $E$, Reychler $H$. éd : De Boeck-Wesmael, Bruxelles, 1991. médecine buccale chirurgie buccale VOL. $8, \mathrm{~N}^{\circ}$ 2002 page 57 